\title{
Preoperative serum albumin as a prognostic factor in patients with upper urinary tract urothelial carcinoma
}

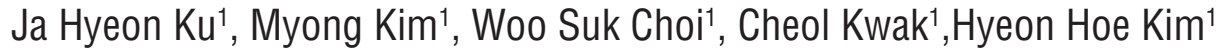 \\ ${ }^{1}$ Department of Urology, Seoul National University College of Medicine, Seoul, Korea
}

\section{ABSTRACT}

Purpose: The study evaluated whether preoperative measures of the C-reactive protein-based systemic inflammatory response may predict cancer survival independent of tumor stage in patients with upper urinary tract urothelial carcinoma (UTUC).

Materials and Methods: Between September 1999 and October 2010, 181 patients submitted to radical nephroureterectomy were available for evaluation. Multivariate survival analyses were performed using Cox's proportional hazards model and the coefficient for each factor was divided by the highest coefficient, multiplied by 4 , and rounded to the nearest integer.

Results: Multivariate analyses showed that tumor location, pathologic T stage, lymphovascular invasion, margin status, and albumin level were independent contributors. The bootstrap-corrected C statistics of the model were 0.813 for disease-specific survival and 0.755 for overall survival, respectively. For time to disease-specific and overall mortality for patients, integrated area under the curve values were 0.792 and 0.739 , respectively. When patients were clustered into three groups according to their model-predicted survival, the 5-year disease-specific survival in the low-, intermediate- and high-risk group was 95.4\%, 76.2\%, and 36.9\%, respectively $(\mathrm{p}<0.001)$, and were $87.8 \%$, $54.4 \%$, and $31.8 \%$, respectively, for overall survival $(\mathrm{p}<0.001)$. Decision curve analysis revealed that the use of model was associated with net benefit gains relative to the treat-all strategy.

Conclusions: Pretreatment albumin is a simple biomarker based on routinely available well-standardized measures, and is not an expensive and time-consuming process. Hypoalbuminemia is an independent marker of poor prognosis in patients with upper urinary tract urothelial carcinoma.

\section{ARTICLE INFO}

\section{Key words:}

Carcinoma, Transitional Cell;

Urinary Tract; Ureter; C-Reactive

Protein; Inflammation

Int Braz J Urol. 2014; 40: 753-62

Submitted for publication:

December 02, 2013

Accepted after revision:

April 06, 2014

\section{INTRODUCTION}

Upper urinary tract urothelial carcinoma (UTUC) is a relatively rare malignancy, accounting for 5\% of all urothelial carcinomas (1). Since these tumors often behave aggressively and local failure rates are high, even after radical surgical treatment, the outcome of patients with UTUC is still generally poor (1). Although tumor stage and histologic grade are the most significant prognos- tic factors for UTUC, the significance of other relevant factors, such as lymphovascular invasion, tumor necrosis, tumor architecture, and concomitant carcinoma in situ, are unclear (2).

Disease outcome in cancer patients is not solely determined by the local characteristics of the tumor. The host systemic inflammatory response (SIR) is also involved (3). As a state of chronic inflammation plays a role in the initiation, promotion, and progression of malignant disease 
(4), host SIR may be an important tumor stage-independent predictor of outcome in cancer. Previous studies have established the prognostic importance of the SIR in patients with localized tumor undergoing curative surgery (5) as well as advanced tumors (6). Based on a previous study, C-reactive protein (CRP) level $>10 \mathrm{mg} / \mathrm{l}$ (7), albumin level $<35 \mathrm{~g} / \mathrm{l}$ (7), and white cell count $>11 \times 10^{9} / 1$ (8) were considered to indicate the presence of a SIR. In particular, CRP-based SIR have been explored and these prognostic scores have been associated with survival in patients with cancer: the combination of serum CRP and albumin as the original Glasgow prognostic score (9) and modified Glasgow prognostic score (10), and a combination of CRP and white cell count in the prognostic index (11).

To our knowledge, a few studies have evaluated the prognostic value of CRP-based SIR in patients with UTUC (12). The aim of this study was to evaluate whether preoperative measures of CRP-based SIR may predict cancer survival, independent of tumor stage, in patients with UTUC.

\section{MATERIALS AND METHODS}

\section{Patient selection and data collection}

The study protocol was approved by our Institutional Review Board. We retrospectively reviewed our database of the 303 patients who underwent radical nephroureterectomy for primary UTUC between September 1999 and October 2010. Exclusion criteria were: 1) no available laboratory data for inflammation, such as CRP, albumin, and white cell count; 2) clinical evidence of infection or inflammatory conditions, such as pneumonia, inflammatory bowel disease, or rheumatoid arthritis; 3) history of bladder tumor at a higher stage than the upper tract disease; 4) preoperative chemotherapy or irradiation; and 5) distant metastatic disease at the time of surgery. In total, 181 patients (median age 65.7 years, range 34.2-90.0 years) were available for evaluation. Median follow-up period of those patients was 56.4 months (range 0.1 to 158.0). The clinical and histologic criteria for these 181 patients are shown in Table- 1 .

\section{Table 1 - Patient characteristics.}

\begin{tabular}{|c|c|}
\hline Variables & \\
\hline Total & 181 \\
\hline Gender (male/female) & $144(79.6) / 37(20.4)$ \\
\hline Age, year (median, interquartile range) & $65.7,59.4-72.6$ \\
\hline Body mass index, $\mathrm{cm} / \mathrm{kg}^{2}$ (median, interquartile range) & $24.1,22.2-25.8$ \\
\hline Tumor location (renal pelvis/ureter/both) & $86(47.5) / 67(37.0) / 28(15.5)$ \\
\hline Pathologic T stage (pTa/is/1/2/3/4) & $22(12.2) / 1(0.6) / 43(23.8) / 35(19.3) / 76(42.0) / 4(2.2)$ \\
\hline Tumor grade $(\mathrm{G} 1 / 2 / 3)$ & $8(4.4) / 113(62.4) / 60(33.1)$ \\
\hline Lymph node stage (Nx/0/+) & $147(81.2) / 26(14.4) / 8(4.4)$ \\
\hline Concomitant carcinoma in situ (absent/present) & $164(90.6) / 17(9.4)$ \\
\hline Lymphovascular invasion (absent/present) & $150(82.9) / 31(17.1)$ \\
\hline Margin status (negative/positive) & $170(93.9) / 11(6.1)$ \\
\hline C-reactive protein, $\mathrm{mg} / \mathrm{l}(\leq 10 />10)$ & $137(75.7) / 44(24.3)$ \\
\hline Albumin, $g / l(\geq 35 /<35)$ & $165(91.2) / 16(8.8)$ \\
\hline White Cell Count, $10^{9} / \mathrm{l}(\leq 11 />11)$ & $168(92.8) / 13(7.2)$ \\
\hline Glasgow Prognostic Score (0/1/2) & $130(71.8) / 42(23.2) / 9(5.0)$ \\
\hline Modified Glasgow Prognostic Score (0/1/2) & $137(75.7) / 35(19.3) / 9(5.0)$ \\
\hline Prognostic Index (0/1/2) & $134(74.0) / 37(20.4) / 10(5.5)$ \\
\hline
\end{tabular}

Data presented are number (\%) or median (interquartile range). 
The kidney, ureter, and bladder cuff were excised en bloc. A regional lymphadenectomy was performed when nodal involvement was suspected from the preoperative workup or was discovered during the procedure. Adjuvant cisplatin-based combination chemotherapy was administered in most patients with disease pT3 or pT4, and/or nodal involvement, but the decision for conducting adjuvant chemotherapy was left to the treating urologists.

\section{Laboratory measurements}

CRP, albumin, and white cell count were measured a few days prior to surgery as part of the routine examination. Routine laboratory measurements including the serum level of CRP, albumin, and white cell count were performed preoperatively. The coefficient of variation for these methods over the range of measurement was $<5 \%$ as established by routine quality control procedures. The prognostic subdivisions used for each described prognostic score are consistent with the previously published literature (Table-2) (7-11).

\section{Pathologic analysis}

All surgical specimens were examined by a dedicated genitourinary pathologist and processed according to standardized procedures. The TNM stage was determined according to the Union Internationale Contre le Cancer/American Joint Cancer Committee 2004 classification. The tumor grade was determined according to the World Health Organization 1973 and 2004 classifications (13). Lymphovascular invasion was defined as the presence of tumor cells in an endothelium-lined space but not in the underlying muscular walls. Tumor multifocality was defined as the synchronous presence of two or more pathologically confirmed tumors in any upper urinary tract location. Positive margin status was defined as tumor presence at linked areas of soft tissue on the surgical specimen.

\section{Postoperative follow-up}

Patients were generally followed every 3-4 months for the first 2 years, every 6 months in years 3 and 4, and annually thereafter. Follow-up examinations included a history, physical examination, blood laboratory tests, urine cyto-
Table 2 - Systemic inflammation-based prognostic scores.

\begin{tabular}{|c|c|}
\hline Prognostic scores & Score \\
\hline \multicolumn{2}{|l|}{ C-reactive protein ${ }^{7}$} \\
\hline$\leq 10 \mathrm{mg} / \mathrm{l}$ & 0 \\
\hline$>10 \mathrm{mg} / \mathrm{l}$ & 1 \\
\hline \multicolumn{2}{|l|}{ Albumin ${ }^{7}$} \\
\hline$\geq 35 \mathrm{~g} / \mathrm{l}$ & 0 \\
\hline$<35 \mathrm{~g} / \mathrm{l}$ & 1 \\
\hline \multicolumn{2}{|l|}{ White cell count ${ }^{8}$} \\
\hline$\leq 11\left(10^{9} / \mathrm{l}\right)$ & 0 \\
\hline$>11\left(10^{9} / \mathrm{I}\right)$ & 1 \\
\hline \multicolumn{2}{|l|}{ Glasgow Prognostic Score ${ }^{9}$} \\
\hline C-reactive protein $\leq 10 \mathrm{mg} / \mathrm{l}$ and albumin $\geq 35 \mathrm{~g} / \mathrm{l}$ & 0 \\
\hline C-reactive protein $\leq 10 \mathrm{mg} / \mathrm{l}$ and albumin $<35 \mathrm{~g} / \mathrm{l}$ & 1 \\
\hline C-reactive protein >10 mg/l & 1 \\
\hline C-reactive protein $>10 \mathrm{mg} / \mathrm{l}$ and albumin $<35 \mathrm{~g} / \mathrm{l}$ & 2 \\
\hline \multicolumn{2}{|l|}{ Modified Glasgow Prognostic Score ${ }^{10}$} \\
\hline C-reactive protein $\leq 10 \mathrm{mg} / \mathrm{l}$ and albumin $\geq 35 \mathrm{~g} / \mathrm{l}$ & 0 \\
\hline C-reactive protein $\leq 10 \mathrm{mg} / \mathrm{l}$ and albumin $<35 \mathrm{~g} / \mathrm{l}$ & 0 \\
\hline C-reactive protein >10 mg/l & 1 \\
\hline C-reactive protein $>10 \mathrm{mg} / \mathrm{l}$ and albumin $<35 \mathrm{~g} / \mathrm{l}$ & 2 \\
\hline \multicolumn{2}{|l|}{ Prognostic Index ${ }^{11}$} \\
\hline $\begin{array}{l}\text { C-reactive protein } \leq 10 \mathrm{mg} / \mathrm{l} \text { and white cell count } \\
\leq 11\left(10^{9} / \mathrm{l}\right)\end{array}$ & 0 \\
\hline $\begin{array}{l}\text { C-reactive protein } \leq 10 \mathrm{mg} / \mathrm{l} \text { and white cell count } \\
>11\left(10^{9} / \mathrm{l}\right)\end{array}$ & 1 \\
\hline $\begin{array}{l}\text { C-reactive protein }>10 \mathrm{mg} / \mathrm{l} \text { and white cell count } \\
\leq 11\left(10^{9} / \mathrm{l}\right)\end{array}$ & 1 \\
\hline $\begin{array}{l}\text { C-reactive protein }>10 \mathrm{mg} / \mathrm{l} \text { and white cell count } \\
>11\left(10^{9} / \mathrm{l}\right)\end{array}$ & 2 \\
\hline
\end{tabular}

logy, cystoscopic evaluation, chest radiography, and abdomino-pelvic computed tomography (CT). Oncological outcome analyses focused on disease-specific survival (DSS). Cause of death was determined by treating physicians, by chart review corroborated by death certificates, or by death certificates alone. Patient data were censored at 
the last follow-up or death if the event of interest had not been attained. All patients who were identified as having died of UTUC had progressive and widely disseminated metastases at the time of death. Patients who died in the perioperative period within 30 days of surgery were censored at time of death for DSS analyses.

\section{Statistical analysis}

Data are presented as median and interquartile range. Several variables were chosen, including gender, patient age, body mass index, pathologic $\mathrm{T}$ stage, tumor grade, lymph node stage, concomitant carcinoma in situ, lymphovascular invasion, margin status, CRP, albumin, white cell count, Glasgow prognostic score, modified Glasgow prognostic score, and prognostic index. Multivariate survival analysis with calculation of hazard ratio (HR) with 95\% confidence interval (CI) was performed using the Cox proportional hazards model including all covariates that were significant on univariate analysis. We applied a weighting method to each variable (14); the coefficient for each factor was divided by the highest coefficient, multiplied by 4 , and rounded to the nearest integer (15). Precision of the reported coefficients was assessed by creating 1,000 bootstrap samples from the entire data set and replicating the estimation process. CIs were obtained using this bootstrap method of the corresponding sampling distributions.

To evaluate the performance of the models, we assessed these models in terms of both discrimination and calibration (16). The discriminative ability of the model was measured quantitatively using the C statistic (17) and time-dependent receiver operating characteristics curve (18). A score was calculated for each patient by adding together the points corresponding to his or her factors, and the patients were divided into three groups on the basis of the score. The survival stratified by risk groups was calculated using the Kaplan-Meier method. Calibration was also assessed by the calibration plot. To determine the net benefit, we relied on decision curve analysis (19).

For all statistical analyses, two-sided $\mathrm{p}<0.05$ was regarded as significant. Analysis was performed using SPSS software (SPSS, Chicago, IL) and R 2.13.0 (R Development Core Team, Vienna, Austria, http://www.R-project.org).

\section{RESULTS}

Table-3 shows the results of the multivariate Cox regression analyses in the cohort related to survival. Univariate analysis revealed that tumor location, pathologic $\mathrm{T}$ stage, tumor grade, lymph node stage, lymphovascular invasion, margin status, and albumin level were significant predictors of disease-specific survival. Multivariate analysis with these variables as covariates showed that tumor location, pathologic T stage, lymphovascular invasion, margin status, and albumin level were independent contributors. The score for DSS was calculated as 2 (if tumors located in both renal pelvis and ureter) +3 (if pT2) or 4 (if $\geq \mathrm{pT} 3$ ) +3 (if lymphovascular invasion) +4 (if positive margin) +2 (if albumin $<35 \mathrm{~g} / \mathrm{l}$ ) and 0 (otherwise). In univariate analysis, tumor location, pathologic T stage, tumor grade, lymph node stage, lymphovascular invasion, margin status, CRP, and albumin level were significantly associated with overall survival (OS). Tumor location, pathologic $\mathrm{T}$ stage, lymphovascular invasion, margin status, and albumin level were independent contributors in the Cox regression model for OS. The score for OS was calculated as 2 (if tumors located in both renal pelvis and ureter) +3 (if $\geq$ pT3) +3 (if lymphovascular invasion) +4 (if positive margin) +3 (if albumin $<35 \mathrm{~g} / \mathrm{l}$ ) and 0 (otherwise) (Table-3).

Model discrimination was good for both models. The bootstrap-corrected C statistics of the multivariate Cox regression model were 0.813 (95\% CI, 0.761-0.865) for DSS and 0.755 (95\% CI, 0.702-0.807) for OS, respectively. To determine the accuracy of DSS and OS by the Cox regression model over the course of a follow-up period, we completed a concordance summary (integrated area under the curve). For time to disease-specific and overall mortality for patients, integrated area under the curve values were 0.792 and 0.739 , respectively (Figure-1). Figure-2 illustrates the Kaplan-Meier curves for patients stratified into three groups from the models. Patients were clustered into three groups according to their model- 


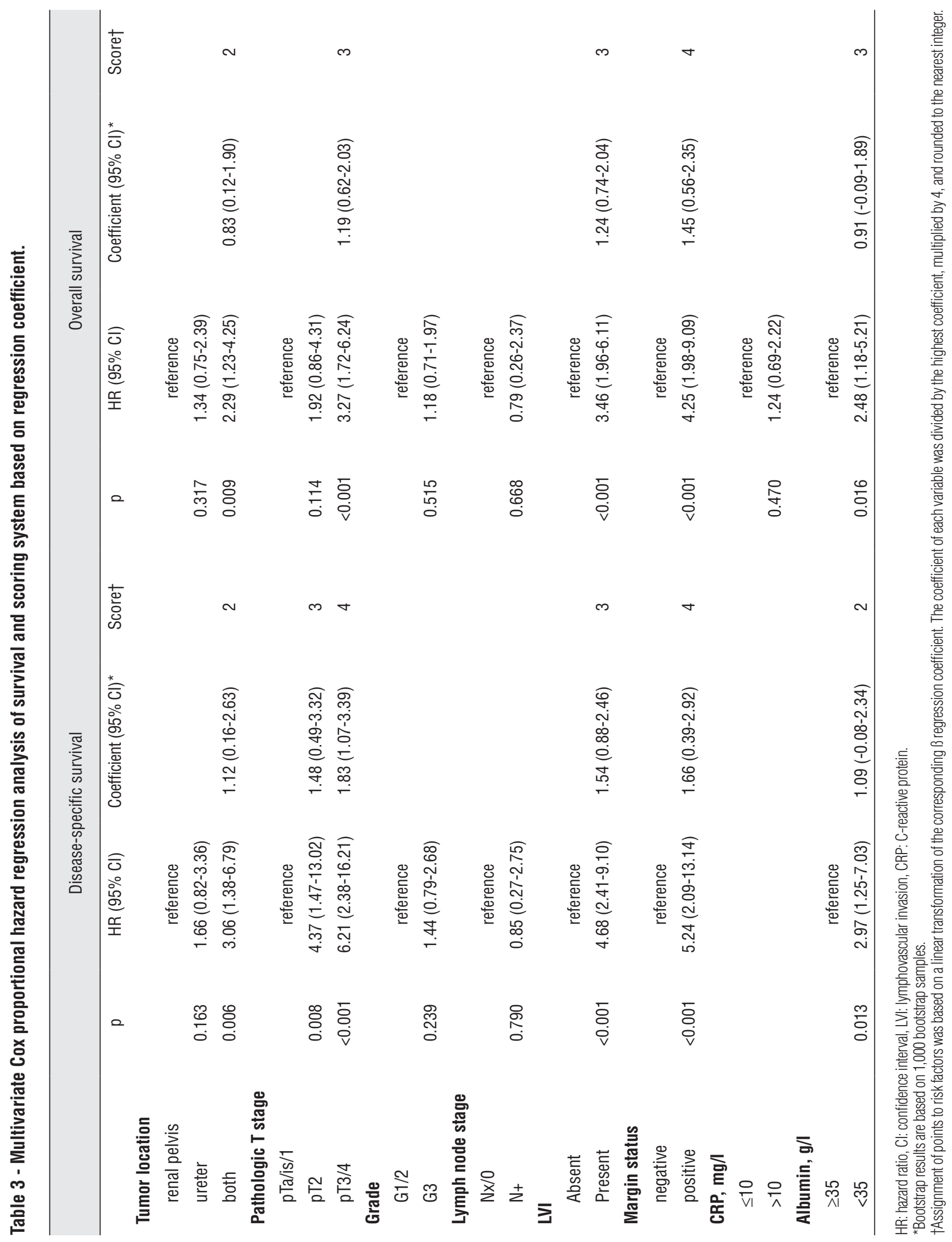


Figure 1 - Time-dependent receiver operating characteristics curve analysis of the Cox regression model. Curve plots the area under the curve over time. (A) Disease-specific survival. Integrated AUC value was 0.792. (B) Overall survival. Integrated AUC value was 0.739 .
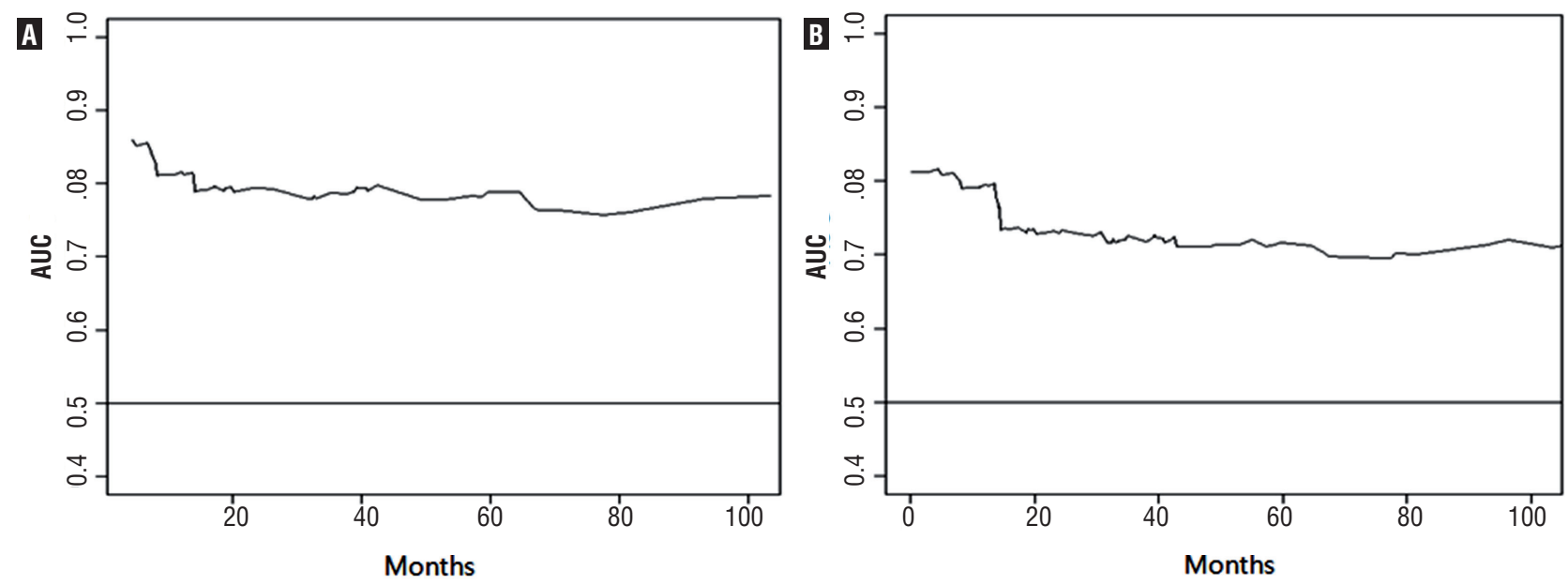

Figure 2 - (A) Kaplan-Meier plots of disease-specific survival of the Cox regression mode $(p<0.0001)$. (B) Kaplan-Meier plots of overall survival of the Cox regression mode $(p<0.0001)$.

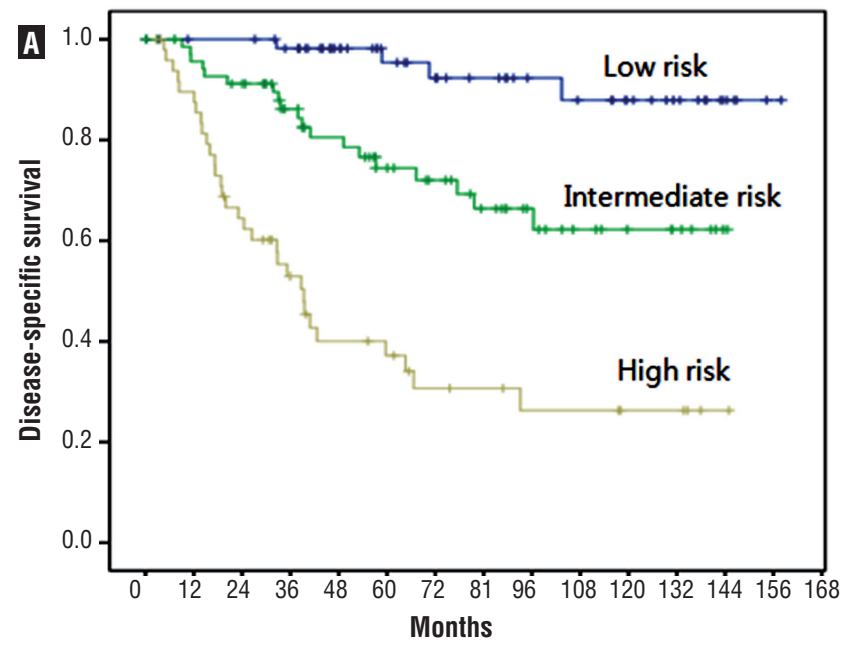

-predicted survival (first quartile, <33\%; second quartile, 33\% to 66\%; third quartile, $>66 \%$ ). As depicted, models discriminated well and log-rank test were all highly significant $(\mathrm{p}<0.001)$. According to the models, the 5-year DSS in the low-, intermediate-, and high-risk group was 95.4\%, $76.2 \%$, and $36.9 \%$, respectively (Figure-2A), and $87.8 \%, 54.4 \%$, and $31.8 \%$, respectively, for OS (Figure-2B). To assess the agreement between the predicted and actual outcomes, we generated calibration curves. The dashed line represents the performance of an ideal model, where the pre-

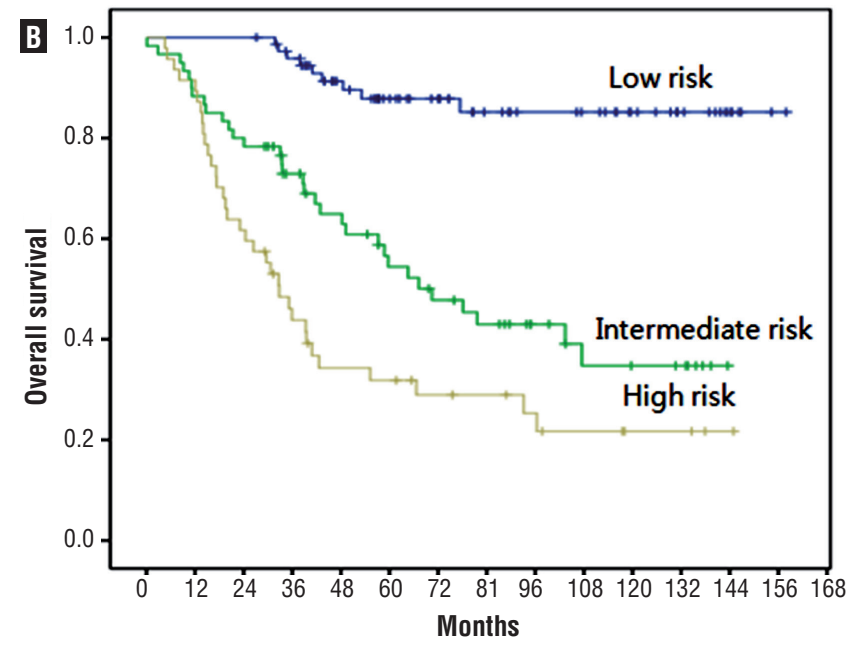

dicted outcome would correspond perfectly with the actual outcome. The performance of model is plotted as the solid line. The models were reasonably calibrated (Figure-3). Figure-4 presents the results of the decision curve analysis at 5 years. In our cohort, the predictions resulted in good net benefit across the range of risk thresholds. Decision curve analysis revealed that the use of model was associated with net benefit gains relative to the treat-all strategy. The models performed well across a wide range of threshold probabilities using decision curve analysis. 
Figure 3 - (A) Calibration curves for 5-year disease-specific survival predictions. (B)
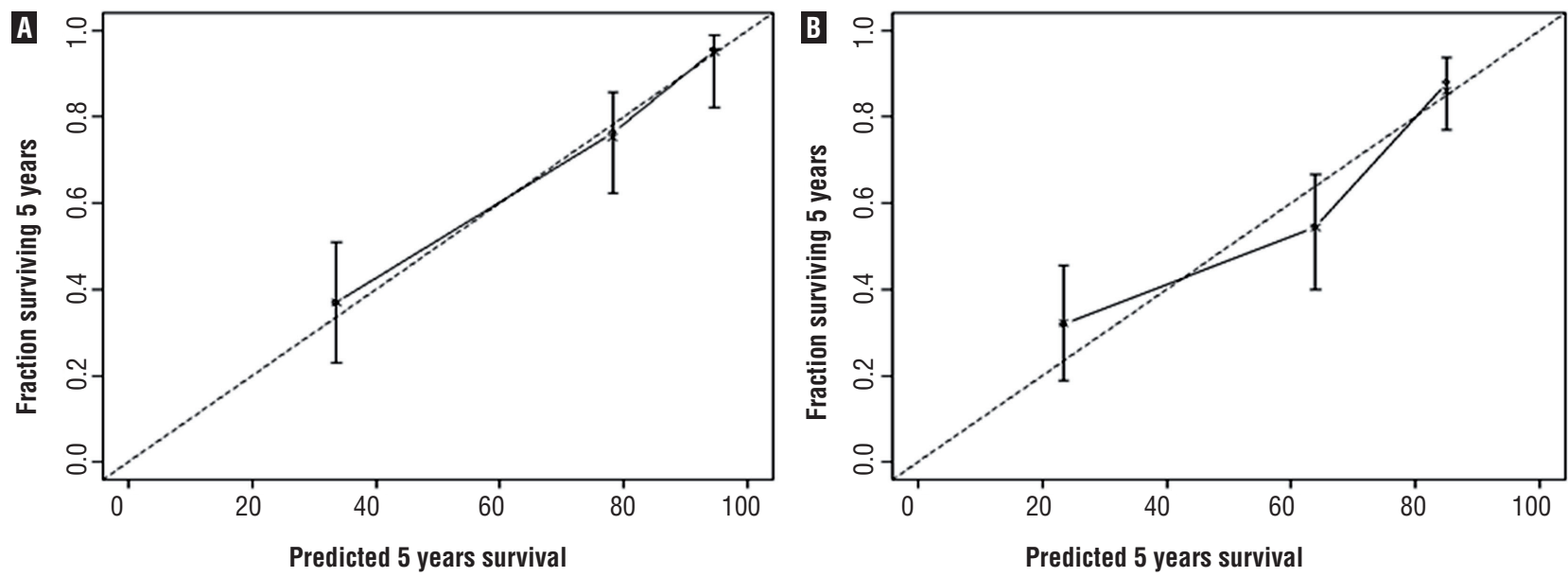

Figure 4 - Decision curve analysis for 5-year survival predictions. (A) Disease-specific survival. (B) Overall survival. In decision curve analysis, the $y$-axis measures net benefit, calculated by summing the benefits (true positives) and subtracting the harms (false positives). The straight line represents the assumption that all patients will die and the horizontal line represents the assumption that no patients will die. The dotted line indicates the net benefit of using the new model.

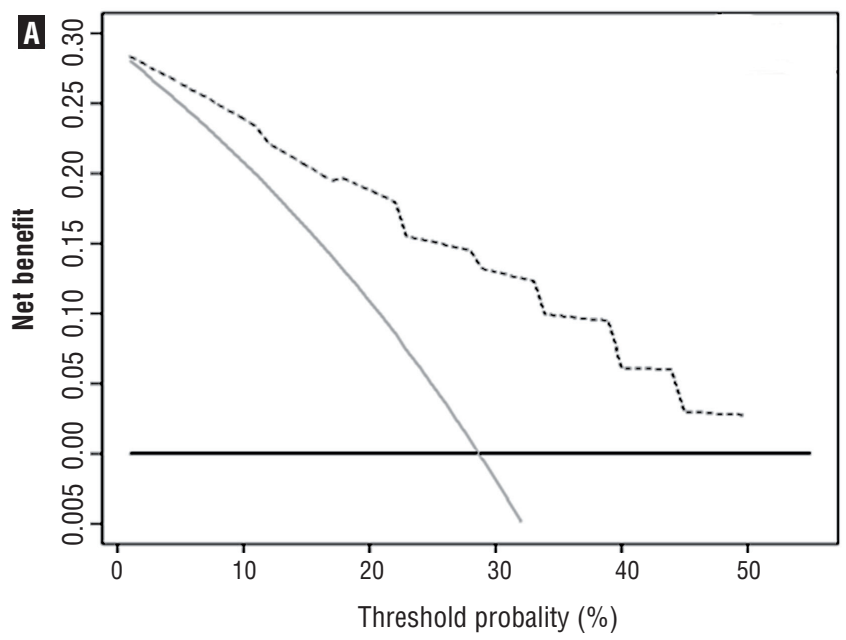

None

All

Model 1

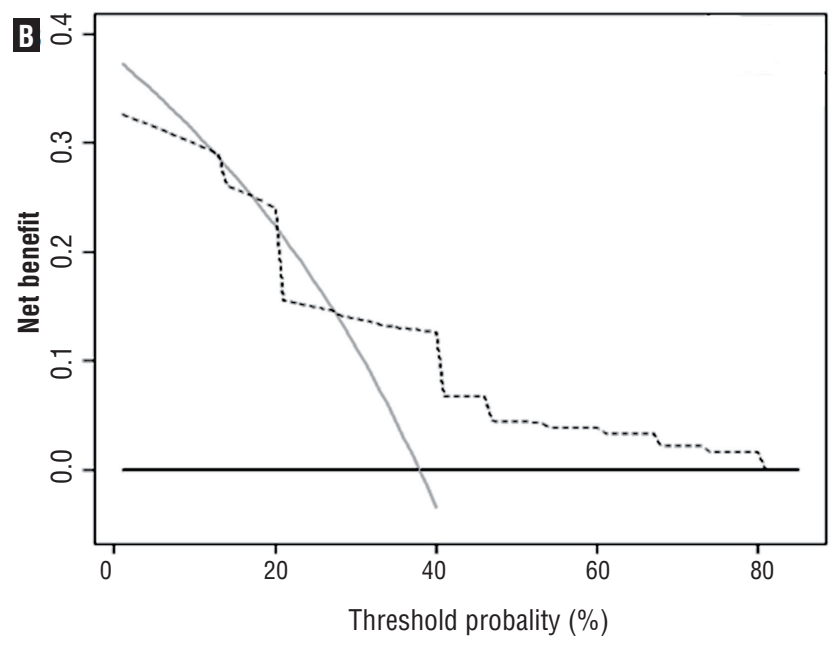

None

All

Model 1

\section{DISCUSSION}

The prognosis of patients with UTUC remains unsatisfactory because it is one of the most aggressive carcinomas. Knowledge about prognosis is essential for patients and clinicians whenever

multimodal treatment is considered. At present, no biomarker has been applicable for predicting the outcomes in patients with UTUC. There is increasing evidence that the presence of SIR is associated with poor outcome in patients undergoing resection for a variety of common solid tumors. 
It has been proposed that there are intrinsic pathways involved in cancer-related inflammation, such as the induction of genetic instability by inflammatory mediators. A recent review proposed that cancer-related inflammation is the seventh hallmark of cancer (3). Inflammation in the tumor microenvironment plays an important role in tumor proliferation and the survival of malignant cells, and promotes tumor angiogenesis, invasion, and metastasis (4).

Recent definitions of cancer cachexia include elevation of CRP and hypoalbuminemia (20). CRP is an acute phase reactant that acts as a surveillance molecule for the activation of the adaptive immune system. It is considered a nonspecific but sensitive marker of systemic inflammation and has been related to most of the signs of nutritional depletion (21). Albumin represents a negative acute phase protein and its level decreases as CRP rises (22). Albumin is no longer considered as a nutritional marker, but rather is considered an inflammatory response marker (23). Increased catabolism, chronic malnutrition, and a chronic inflammatory reaction due to cancer eventually lead to hypoalbuminemia. Hypoalbuminemia may be due to the production of cytokines, such as interleukin-6, which modulates the production of albumin by hepatocytes (24). Alternatively, tumor necrosis factor may increase the permeability of the microvasculature, allowing an increased transcapillary passage of albumin (25). Also, the presence of micrometastatic tumor cells in liver may induce Kuppfer cells to produce a variety of cytokines, which may modulate albumin synthesis by hepatocytes $(24,26)$.

Hypoalbuminemia provides additional prognostic information for patients with cancer in various organs. Hypoalbuminemia is predictive of increased 90-day mortality and poor overall survival after radical cystectomy in patients with bladder cancer (27). In patients with hepatocellular carcinoma, hypoalbuminemia is an independent poor prognostic factor (28). Separate studies reported that preoperative hypoalbuminemia is associated with earlier tumor recurrence and death following potentially curative resection for colon and rectal cancer $(24,29)$. Hypoalbuminemia as a marker of the SIR and not CRP is a significant and independent predictor of survival in patients with primary operable breast cancer (30).

It is not yet established if any specific component of SIR is a better predictor of cancer survival. This study is the first to show hypoalbuminemia to be superior to other CRP-based SIR prognostic scores for the prediction of prognosis in patients with UTUC. Presently, among the inflammation-based prognostic scores, only albumin was significantly associated with disease-specific survival and had prognostic value independent of TNM stage. This would suggest that albumin has superior prognostic value than other scores based on CRP (Glasgow prognostic score, modified Glasgow prognostic score, and prognostic index) in terms of outcome and should be used in preference to cellular markers of the SIR. Therefore, any further development of prognostic scores based on the SIR should include albumin. However, since the basis of the relationship between the SIR and poorer DSS in patients with UTUC is not clear, the cause-effect relationship between a SIR and DSS is questionable.

We used a wide array of tools to evaluate our multivariate Cox regression model including discrimination as well as calibration analyses. In addition, we used decision curve analysis to show the clinical net benefit. Our study results support the view that hypoalbuminemia has significant prognostic value. We observed a good prediction of clinical outcome by the model. The accuracy of the model for DSS and OS disease-specific and overall survival was $81.3 \%$ and $75.5 \%$, respectively. The Kaplan-Meier curve was within the boundaries of predictions for each of the three strata. Using decision curve analysis, the model demonstrated net benefit gains for predictions of the examined end points at 5 years after radical nephroureterectomy.

The present cohort has a number of limitations. Despite the retrospective nature of the data collection, not all patients in our database had preoperative data on CRP-based SIR. Therefore, the patients were not necessarily representative of all patients with UTUC in general. A large-scale prospective validation study is needed to confirm the results.

However, despite the retrospective nature of the study and a relatively small number of pa- 
tients in a single center, a homogeneous patient population was sufficient to indicate the significance of hypoalbuminemia as a postoperative prognostication of patients with UTUC.

\section{CONCLUSIONS}

In the present study, hypoalbuminemia predicted DSS as well as OS independent of stage in patients with UTUC. Pretreatment albumin is a simple biomarker based on routinely available well-standardized measures, is not expensive, and does not involve a time-consuming process. Our study demonstrates that hypoalbuminemia is an independent marker of poor prognosis in patients with UTUC and is superior to other CRP-based SIR protocols in terms of prognostic ability.

\section{ABBREVIATIONS}

$\mathrm{CI}=$ confidence interval

$\mathrm{CRP}=\mathrm{C}$-reactive protein

$\mathrm{CT}=$ computed tomography

DSS $=$ disease-specific survival

$\mathrm{HR}=$ hazard ratio

OS =overall survival

SIR = systemic inflammatory response

UTUC = upper urinary tract urothelial carcinoma

\section{REFERENCES}

1. Jemal A, Siegel R, Ward E, Hao Y, Xu J, Thun MJ: Cancer statistics, 2009. CA Cancer J Clin. 2009; 59: 225-49.

2. Rouprêt M, Zigeuner R, Palou J, Boehle A, Kaasinen E, Sylvester $\mathrm{R}$, et al.: European guidelines for the diagnosis and management of upper urinary tract urothelial cell carcinomas: 2011 update. Eur Urol. 2011; 59: 584-94.

3. Colotta F, Allavena P, Sica A, Garlanda C, Mantovani A: Cancerrelated inflammation, the seventh hallmark of cancer: links to genetic instability. Carcinogenesis. 2009; 30: 1073-81.

4. Mantovani A, Allavena P, Sica A, Balkwill F: Cancer-related inflammation. Nature. 2008; 454: 436-44.

5. Lamb GW, Aitchison M, Ramsey S, Housley SL, McMillan DC: Clinical utility of the Glasgow Prognostic Score in patients undergoing curative nephrectomy for renal clear cell cancer: basis of new prognostic scoring systems. $\mathrm{Br} J$ Cancer. 2012; 106: 279-83.
6. Sharma R, Hook J, Kumar M, Gabra H: Evaluation of an inflammation-based prognostic score in patients with advanced ovarian cancer. Eur J Cancer. 2008; 44: 251-6.

7. Glen P, Jamieson NB, McMillan DC, Carter R, Imrie CW, McKay CJ: Evaluation of an inflammation-based prognostic score in patients with inoperable pancreatic cancer. Pancreatology. 2006; 6: 450-3.

8. Proctor MJ, Morrison DS, Talwar D, Balmer SM, Fletcher CD, O'Reilly DS, et al.: A comparison of inflammationbased prognostic scores in patients with cancer. A Glasgow Inflammation Outcome Study. Eur J Cancer. 2011; 47: 263341.

9. Forrest LM, McMillan DC, McArdle CS, Angerson WJ, Dunlop DJ: Evaluation of cumulative prognostic scores based on the systemic inflammatory response in patients with inoperable non-small-cell lung cancer. Br J Cancer. 2003; 89: 1028-30.

10. McMillan DC: An inflammation-based prognostic score and its role in the nutrition-based management of patients with cancer. Proc Nutr Soc. 2008; 67: 257-62.

11. Kasymjanova G, MacDonald N, Agulnik JS, Cohen V, Pepe $\mathrm{C}$, Kreisman $\mathrm{H}$, et al.: The predictive value of pre-treatment inflammatory markers in advanced non-small-cell lung cancer. Curr Oncol. 2010; 17: 52-8.

12. Saito K, Kawakami S, Ohtsuka Y, Fujii Y, Masuda H, Kumagai $\mathrm{J}$, et al.: The impact of preoperative serum C-reactive protein on the prognosis of patients with upper urinary tract urothelial carcinoma treated surgically. BJU Int. 2007; 100: 269-73.

13. Lopez-Beltran A, Sauter G, Gasser T: Tumors of the urinary system: infiltrating urothelial carcinoma. In: Eble JN, Sauter G, Epstein JI (ed.), World Health Organization Classification of Tumours-Tumours of the Urinary System and Male Genital Organs. Lyon, IARC Press. 2004; vol 1, pp. 93-109.

14. Rassi A Jr, Rassi A, Little WC, Xavier SS, Rassi SG, Rassi $A G$, et al.: Development and validation of a risk score for predicting death in Chagas' heart disease. N Engl J Med. 2006; 355: 799-808.

15. limura Y, Saito K, Fujii Y, Kumagai J, Kawakami S, Komai Y, et al.: Development and external validation of a new outcome prediction model for patients with clear cell renal cell carcinoma treated with nephrectomy based on preoperative serum C-reactive protein and TNM classification: the TNM-C score. J Urol. 2009; 181: 1004-12; discussion 1012.

16. Altman DG, Royston $P$ : What do we mean by validating a prognostic model? Stat Med. 2000 Feb 29; 19: 453-73.

17. Harrell FE Jr, Lee KL, Mark DB: Multivariable prognostic models: issues in developing models, evaluating assumptions and adequacy, and measuring and reducing errors. Stat Med. 1996; 15: 361-87.

18. Heagerty PJ, Lumley T, Pepe MS: Time-dependent ROC curves for censored survival data and a diagnostic marker. Biometrics. 2000; 56: 337-44. 
19. Vickers AJ, Elkin EB: Decision curve analysis: a novel method for evaluating prediction models. Med Decis Making. 2006; 26: 565-74.

20. Fearon KC, Voss AC, Hustead DS; Cancer Cachexia Study Group: Definition of cancer cachexia: effect of weight loss, reduced food intake, and systemic inflammation on functional status and prognosis. Am J Clin Nutr. 2006; 83: 1345-50.

21. Mahmoud FA, Rivera NI: The role of C-reactive protein as a prognostic indicator in advanced cancer. Curr Oncol Rep. 2002; 4: 250-5

22. McMillan DC: Systemic inflammation, nutritional status and survival in patients with cancer. Curr Opin Clin Nutr Metab Care. 2009; 12: 223-6.

23. Barbosa-Silva MC: Subjective and objective nutritional assessment methods: what do they really assess? Curr Opin Clin Nutr Metab Care. 2008; 11: 248-54.

24. Heys SD, Walker LG, Deehan DJ, Eremin OE: Serum albumin: a prognostic indicator in patients with colorectal cancer. J R Coll Surg Edinb. 1998; 43: 163-8.

25. Deehan DJ, Heys SD, Simpson WG, Broom J, Franks C, Eremin 0: In vivo cytokine production and recombinant interleukin 2 immunotherapy: an insight into the possible mechanisms underlying clinical responses. $\mathrm{Br} \mathrm{J}$ Cancer. 1994; 69: 1130-5.

26. Fearon KC, Falconer JS, Slater C, McMillan DC, Ross JA, Preston $\mathrm{T}$ : Albumin synthesis rates are not decreased in hypoalbuminemic cachectic cancer patients with an ongoing acute-phase protein response. Ann Surg. 1998;227:249-54.
27. Gregg JR, Cookson MS, Phillips S, Salem S, Chang SS, Clark PE, et al.: Effect of preoperative nutritional deficiency on mortality after radical cystectomy for bladder cancer. $\mathrm{J}$ Urol. 2011; 185: 90-6.

28. Cho YK, Chung JW, Kim JK, Ahn YS, Kim MY, Park YO, et al.: Comparison of 7 staging systems for patients with hepatocellular carcinoma undergoing transarterial chemoembolization. Cancer. 2008; 112: 352-61.

29. Cengiz 0, Kocer B, Sürmeli S, Santicky MJ, Soran A: Are pretreatment serum albumin and cholesterol levels prognostic tools in patients with colorectal carcinoma? Med Sci Monit. 2006; 12: CR240-7.

30. Al Murri AM, Doughty JC, Lannigan A, Wilson C, McArdle CS, et al.: The relationship between deprivation, tumour stage and the systemic inflammatory response in patients with primary operable breast cancer. $\mathrm{Br} \mathrm{J}$ Cancer. 2004; 91: 1063-5.

Correspondence address: Cheol Kwak, MD, PhD Department of Urology, Seoul National University Hospital, 101 Daehak-ro, Jongno-gu, Seoul 110-744, Korea

Fax: +82 2 742-4665

E-mail: mdrafael@snu.ac.kr 